\title{
Verbal Memory
}

National Cancer Institute

\section{Source}

National Cancer Institute. Verbal Memory. NCI Thesaurus. Code C88416.

The ability to store and recall linguistic information. 